\title{
Phenytoin accelerates tendon healing in a rat model of Achilles tendon rupture
}

\author{
Hajipour B ${ }^{1}$, Navali $\mathrm{AM}^{2}$, Ali Mohammad Zadeh Taher $\mathrm{S}^{3}$, Mousavi $\mathrm{G}^{4}$, Gahvechi Akbari $\mathrm{M}^{5}$, \\ Maleki Miyandoab $\mathrm{T}^{1}$, Roshangar L ${ }^{6}$, Mohammadi Saleh $\mathrm{B}^{4}$, Asvadi Kermani $\mathrm{T}^{7}$, Moutab Laleh $\mathrm{F}^{4}$, \\ Ghabili $\mathrm{M}^{8}$
}

Department of Surgery, Urmia University of Medical Sciences, Urmia, Iran, and Army University of Medical Sciences, Tehran, Iran, Student Research Committee, Urmia University of Medical Sciences, Urmia, Iran. am.navali@yahoo.com

\begin{abstract}
INTRODUCTION: Tendons are vulnerable to various types of acute or chronic injures. Different methods have been investigated to achieve better healing. Phenytoin is a drug which could stimulate fibroblasts to produce collagen. This experimental study was performed to assess the effect of phenytoin on tendon healing in a rat model of tendon rupture.

METHODS: Thirty healthy rats were divided into 3 groups, 1) Sham group; 2) Tendon rupture; 3) Tendon rupture+phenytoin (100 mg/kg intraperitoneally) for 21 days. On 21st day after tendon injury, the rats were anesthetized and tendon tissue was sampled for studying by light and electron microscopy.

RESULTS: Qualitative and quantitative microscopic comparisons of the repair tissues of both groups were made on the 21st day. The results obtained from light and electron microscopy studies showed that tendon tissue healing was significantly better in phenytoin group compared to the control group $(p<0.05)$.

CONCLUSIONS: Systemic administration of phenytoin may have a positive effect on tendon healing by increasing fibroblast quantity, fibrillar collagen synthesis, vascularity, and suppressing inflammation (Tab. 2, Ref. 25). Text in PDF www.elis.sk.

KEY WORDS: tendon, healing, phenytoin, rat.
\end{abstract}

\section{Introduction}

Achilles tendon rupture is a relatively common injury resulting in a prolonged healing process and significant disability. The injury associated with Achilles tendon rupture is sometimes a result of athletic activity and middle-aged individuals are particularly prone to the injury (1).

Tendons are composed of parallel bundles of collagen fibers (primarily type I) and rows of fibroblasts situated between these bundles. The most important function of the type I collagen, the main functional component of tendons, is to provide resistance to force, tension and stretch (2). The Achilles tendon, formed by the merging of gastrocnemius and soleus tendons, is the thickest and

${ }^{1}$ Department of Surgery, Urmia University of Medical Sciences, Urmia, Iran, and Army University of Medical Sciences, Tehran, Iran, Student Research Committee, Urmia University of Medical Sciences, Urmia, Iran, ${ }^{2}$ Department of Orthopedics, Tabriz University of Medical Sciences, Tabriz, Iran, ${ }^{3}$ Department of Orthopedics, Army University of Medical Sciences, Tehran, Iran, ${ }^{4}$ Department of Clinical Sciences, Faculty of Veterinary Sciences, Tabriz Branch, Islamic Azad University, Tabriz, Iran, ${ }^{5}$ Department of Physical Medicine and Rehabilitation, Tehran University of Medical Sciences, Tehran, Iran, ${ }^{6}$ Department of Histology, Tabriz University of Medical Sciences, Tabriz, Iran, ${ }^{7}$ Department of Surgery, Tabriz University of Medical Sciences, Tabriz, Iran, and ${ }^{8}$ Medical Philosophy and History Research Center, Tabriz University of Medical Sciences, Tabriz, Iran

Address forcorrespondence: AM Navali, MD, Department of Orthopedics, Tabriz University of Medical Sciences, Tabriz, Iran strongest tendon in the human body. Achilles tendon rupture is still a challenging problem, and the peak annual incidence of Achilles tendon rupture has been reported as 18 per 100,000 (3). The course of the tendon healing depends on several factors such as age, nutrition, systemic diseases, hormones, infections and vitamins (4).

The tendon is a hypocellular and hypovascular tissue that heals slowly and usually repairs through scar formation. The healing process may last for several months and even years, and injured tendons never recover the functional properties of healthy and normal tendons (5). In an attempt to compensate for the inefficient repair process, researchers and clinicians have developed and tested various treatment strategies including nonsteroidal anti-inflammatory drugs (NSAIDs), tissue engineering, mobilization, mechanical loading, and other physical modalities such as ultrasound, extracorporeal shock-wave therapy and electrical stimulation (5).

Phenytoin (sodium diphenylhydantoin) is a well-known antiepileptic medication that has been used clinically since 1938 . Phenytoin can stimulate responsive sub-populations of fibroblasts to synthesize increased amounts of collagen and glycosaminoglycans (6) and has been used in the healing of pressure sores, venous stasis, diabetic ulcers, traumatic wounds, and burns (7). It has been postulated that phenytoin administration might possibly promote wound healing by increasing the collagen deposition in rat skin. Although exact tissue mechanisms of action of phenytoin are unknown, growth factors, especially vascular endothelial 
growth factor (VEGE) and basic fibroblast growth factor (BFGF), have been indicated as mediators for the neovascularization and collagen fiber production by the fibroblasts; important steps for the wound healing (8).

To the best of our knowledge, possible role of phenytoin in tendon healing process has not been hitherto investigated in the literature. Therefore, the aim of the present study was to evaluate the effect of phenytoin on tendon healing in a rat model of tendon rupture.

\section{Methods}

\section{Animals}

Thirty male Wistar rats (6-8 weeks, average body weight of 270 g) were randomly divided into 3 groups. In Group 1 (sham group), animals were anesthetized and posterior incision was performed on the skin, superior to the Achilles tendon. In Group 2 animals underwent anesthesia and Achilles tendon rupture. In Group 3, animals underwent tendon rupture and also received 100 $\mathrm{mg} / \mathrm{kg}$ phenytoin intraperitoneally for 21 days (9). The animals were kept and treated according to international guidelines for the care of laboratory animals.

\section{Surgery protocol}

Under general anesthesia, a posterior incision was made to expose the patellar tendon. The Achilles and plantaris tendons were stripped from the surrounding fascia. The Achilles tendon was transected five millimeters proximal to its insertion to the calcaneus. The plantaris tendon was also transected to prevent internal splint effect. The Achilles tendon was repaired by using $6 / 0$ ethilon monofilament nylon sutures. The wound was closed with $3 / 0$ ethilon monofilament uninterrupted sutures (10). The rats were allowed free cage movement immediately after surgery. At day 21 after injury, the rats were sacrificed. The Achilles tendons were harvested, washed in phosphate buffered saline (PBS), fixed in buffered formalin, and then used to prepare 5-mm-thick histological sections for hematoxylin and eosin staining. Achilles tendons without injury were used as an ideal end point of tendon healing and control.

\section{Light microscopy study}

Tissue samples were fixed in $10 \%$ neutral buffered formalin overnight, and then dehydrated with alcohol. The fixed tissue was processed, embedded in paraffin, and sectioned at 3 micrometers. Finally, tissue sections were stained with haematoxylin and eosin. Fibroblast density, inflammation, vascularity and collagen fiber orientation were graded on a four-point grading system on the following scale: 0 (normal appearance), 1 (slightly abnormal appearance), 2 (moderately abnormal appearance), and 3 (markedly abnormal appearance) (11). To investigate the collagen fiber orientation, Masson-Trichrome stain was used. For each staining technique, two slides were prepared. The area of specimen showing the most advanced pathological changes was selected, and the worst possible results for each slide were used in this study. Slides were examined by the same pathologist, who was blinded to the study protocol, under light microscope (Olympus Bx50 Shinjuku, Monolith, Tokyo, Japan) (10).

\section{Electron microscopy preparation}

For transmission electron microscopy, the tendon biopsies from rats of all three studied groups were cut into $1 \mathrm{~mm} \times 1 \mathrm{~mm}$ and fixed in $2 \%$ glutaraldehyde in a $0.1 \mathrm{M}$ phosphate buffer and postfixed in $1 \%$ aqueous osmium tetroxide. The pieces were then dehydrated through graded concentration of ethanol, and embedded in resin. One micron semi-thin sections were stained with tolidine blue. Ultra-thin sections from selected blocks were stained with uranyl acetate and lead citrate and observed in a LEO 906 type transmission electron microscope (Carl Zeiss, Oberkochen, Germany).

\section{Statistical analysis}

Data were presented as mean \pm standard deviation (SD). Histological sum grades between the groups were analyzed using Mann-Whitney U test. Statistical analysis was performed with SPSS for windows version 16.0 (SPSS Inc, Chicago, IL, USA). A $\mathrm{p}$ value $\leq 0.05$ was considered statistically significant.

\section{Results}

\section{Light microscopy results}

Data showed that fibroblast density was higher in both tendon injury and tendon injury + phenytoin groups compared to the sham group $(\mathrm{p}=0.008 \mathrm{vs} \mathrm{p}=0.0001)$. On the other hand, the fibroblast density was significantly higher in tendon injury + phenytoin group than tendon injury group $(\mathrm{p}=0.002)$ (Tabs 1 and 2$)$.

Inflammatory score was significantly higher in the tendon injury group than in the sham group $(\mathrm{p}=0.001)$ and tendon injury + phenytoin group $(\mathrm{p}=0.009)$. However, there was no statistical difference in inflammatory score between the sham and tendon injury + phenytoin groups $(\mathrm{p}=0.156)($ Tabs 1 and 2$)$.

The mean histological grade of collagen fiber abnormality was significantly greater in tendon injury group than sham group ( $p$ $=0.005)$ and tendon injury + phenytoin group $(p=0.001)$. In ad-

Tab. 1. Distribution of tendon injury grades.

\begin{tabular}{|c|c|c|c|c|c|c|c|c|c|c|c|c|c|c|c|c|}
\hline & \multicolumn{4}{|c|}{ Fibroblast } & \multicolumn{4}{|c|}{ Inflammation } & \multicolumn{4}{|c|}{ Vasculogenesis } & \multicolumn{4}{|c|}{ Collagen } \\
\hline & 0 & 1 & 2 & 3 & 0 & 1 & 2 & 3 & 0 & 1 & 2 & 3 & 0 & 1 & 2 & 3 \\
\hline Sham & 8 & 2 & 0 & 0 & 7 & 3 & 0 & 0 & 9 & 1 & 0 & 0 & 9 & 1 & 0 & 0 \\
\hline Tendon injury & 2 & 7 & 1 & 0 & 0 & 2 & 4 & 4 & 3 & 4 & 3 & 0 & 0 & 1 & 3 & 6 \\
\hline Tendon injury + Phenytoin & 0 & 2 & 5 & 3 & 4 & 5 & 1 & 0 & 0 & 4 & 6 & 0 & 2 & 6 & 2 & 0 \\
\hline
\end{tabular}

Fibroblast density, inflammation, vascularity and collagen fiber orientation were graded on a four-point grading system on the following scale: 0 (normal appearance), 1 (slightly abnormal appearance), 2 (moderately abnormal appearance), and 3 (markedly abnormal appearance). 
Tab. 2. Summary of the mean histological sum grade.

\begin{tabular}{lccc}
\hline & Fibroblast & Inflammation & Vasculogenesis \\
\hline Sham & $0.20 \pm 0.42$ & $0.30 \pm 0.48$ & $0.10 \pm 0.31$ \\
Tendon injury & $0.90 \pm 0.56$ & $1.90 \pm 0.99$ & $1.00 \pm 0.81$ \\
Tendon injury +Phenytoin & $2.10 \pm 0.73$ & $0.70 \pm 0.67$ & $1.60 \pm 0.51$ \\
\hline
\end{tabular}

Values are given as mean \pm standard deviation. Tendon healing was better in tendon injury + phenytoin group compared to tendon injury group, and phenytoin administration significantly accelerated the tendon healing in rats (fibroblast density $(\mathrm{p}=0.002)$, inflammatory score $(0.009)$, collagen fiber abnormality $(0.001)$ and vasculogenesis $(0.001)$ ).

dition, the mean histological grade of collagen fiber abnormality was significantly higher in tendon injury + phenytoin group than sham group $(\mathrm{p}=0.002)$ (Tabs 1 and 2$)$.

Data showed that vasculogenesis was significantly higher in tendon injury + phenytoin group compared to tendon injury group $(\mathrm{p}=0.001)$. Moreover, vasculogenesis was significantly higher in both tendon injury and tendon injury+ phenytoin groups compared to sham group $(\mathrm{p}=0.005$ and $\mathrm{p}=0.002$, respectively) (Tabs 1 and 2).

\section{Electron microscopy results}

We used the fibrillar collagen formation, collagen arrangement, cellular function and level of cellular synthesis as histopathologic evaluation parameters. Histologically, collagen synthesis was better and more organized in phenytoin+tendon injury group compared to tendon injury group. Significant differences were observed between phenytoin + tendon injury group and tendon injury group. There was marked mitochondrial degeneration and less fibrillar collagen synthesis in tendon injury group. A better healing was observed in phenytoin + tendon injury compared to tendon injury group (control).

\section{Discussion}

The findings of the present study revealed that phenytoin treatment after tendon injury for a period of 21 days enhanced tendon healing, collagen synthesis and vascularity of the injured tendon. Furthermore, our data indicated that inflammation grade was significantly lower in the phenytoin group comparedto control group, implying anti-inflammatory role of phenytoin. To the best of our knowledge, the present study is the first investigation orchestrated to assess the effect of phenytoin on tendon healing in a rat model of tendon rupture. As gingival hyperplasia affects approximately $50 \%$ of patients on phenytoin as an anti-epileptic drug, possible therapeutic role of phenytoin in the wound healing has been deemed. Subsequently, a number of clinical trials have provided evidence of an increased rate of repair with reduced edema and inflammation and enhanced formation of granulation tissue following the topical application of phenytoin to cutaneous wounds (11).

Tendon healing occurs in three overlapping phases. In the initial inflammatory phase, erythrocytes and inflammatory cells, particularly neutrophils, enter the site of injury. In the first 24 hours, monocytes and macrophages predominate, and phagocytosis of necrotic materials occurs. Vasoactive and chemotactic factors are released with increased vascular permeability, initiation of angiogenesis, stimulation of tenocyte proliferation, and recruitment of more inflammatory cells. Tenocytes gradually migrate to the wound, and type III collagen synthesis is initiated. After a few days, the remodeling stage begins during which synthesis of type III collagen peaks, lasting for a few weeks. Water content and glycosaminoglycan concentrations remain high during this stage (12). After approximately 6 weeks, the modeling stage commences and the healing tissue is resized and reshaped. Large quantities of type I collagen are synthesized during early modeling stage. After 10 weeks, the maturation stage occurs, with gradual change of fibrous tissue to scar-like tendon tissue over the course of one year (13). The management of tendon injury is a challenge for physicians. Therefore, factors affecting the tendon healing are extremely significant (14).

The possible mechanism of action by which phenytoin promotes wound healing has been investigated. Various in vitro and clinical studies have indicated that phenytoin increases the proliferation of fibroblasts (15), collagen deposition (16), neovascularisation (17), and the granulation tissue formation (18). In our investigation, phenytoin administration increased the fibroblast accumulation at the injury site; the fibroblast index was about 2.5 times higher in the phenytoin group comparedto the control group. Our data showed that collagen grade and collagen arrangement were higher in tendon injury + phenytoin group compared to tendon injury group. Moreover, phenytoin administration increased collagen density at the injury site. Increased collagen may be due to the effect of phenytoin on transcription rates of the collagen genes or posttranslational mRNA processing (19). Phenytoin has also been reported to enhance the maturation of collagen in both normal skin and granulation tissue in rats, possibly by promoting collagen cross-linking (19). Eisenberg et al determined that the increased collagenization with phenytoin treatment was due to the inhibition of collagenase (20). Nevertheless, Bauer and colleagues found that phenytoin did not inhibit the enzyme directly, but decreased its synthesis and/or secretion (21). Swamy et al reported that phenytoin, as wound healing agent, had potent growth factors inducing activity. The most interesting finding in their study was that phenytoin accelerated the autocrine and paracrine activities of growth factors by up-regulating the related receptors (22). It has also been shown that phenytoin induces both latent TGFb and PDGF and also up-regulates the various matrix remodeling genes and receptors at the mRNA level in vitro, possibly implying its role in promoting angiogenesis, collagen synthesis and thus tendon repair (23-25).

\section{Conclusion}

Phenytoin administration may enhance tendon healing by suppressing inflammation, recruiting fibroblasts into the injury site, in- 
543-546

ducing vasculogenesis, collagen formation, collagen arrangement, cellular function and protecting mitochondria degeneration in ruptured tendon in rat. Regarding slow healing of tendon after injury, phenytoin may be a useful agent for accelerating the tendon healing process. Although our study demonstrates that the administration of phenytoin to rats leads to significantly improved tendon healing, mechanism of action of phenytoin in this process still remains obscure. Nonetheless, these effects of phenytoin may be attributed to the growth factors. More investigations are required to further delineate the effects of phenytoin on the tendon-healing process.

\section{References}

1. Suckow MA, Hodde JP, Wolter WR, Hiles MC. Repair of experimental Achilles tenotomy with porcine renal capsule material in a rat model. J Mater Sci Mater Med 2007; 18: 1105-1110.

2. James R, Kesturu G, Balian G, Chhabra AB. Tendon: biology, biomechanics, repair, growth factors, and evolving treatment options. J Hand Surg Am 2008; 33: 102-112.

3. Leppilahti J, Puranen J, Orava S. Incidence of Achilles tendon rupture. Acta Orthop Scand 1996; 67: 277-279.

4. Omeroğlu S, Peker T, Türközkan N, Omeroğlu H. High-dose vitamin $\mathrm{C}$ supplementation accelerates the Achilles tendon healing in healthy rats. Arch Orthop Trauma Surg 2009; 129: 281-286.

5. Lin TW, Cardenas L, Soslowsky LJ. Biomechanics of tendon injury and repair. J Biomech 2004; 37: 865-877.

6. Vernillo AT, Schwartz NB. The effects of phenytoin (5,5-diphenylhydantoin) on human gingival fibroblasts in culture. J Periodontal Res 1987; 22: 307-312.

7. Talas G, Brown RA, McGrouther DA. Role of phenytoin in wound healing a wound pharmacology perspective. Biochem Pharmacol 1999; 57: $1085-1094$.

8. Turan M, Saraydýn SU, Bulut HE, Elagöz S, Cetinkaya O, Karadayi K, Canbay E, Sen M. Do vascular endothelial growth factor and basic fibroblast growth factor promote phenytoin's wound healing effect in rat? An immunohistochemical and histopathologic study. Dermatol Surg 2004; 30: 1303-1309.

9. Zhou X, Li YM, Ji WJ, Jiang TM, Sun XN, Zhu Y, Shi R. Phenytoin can accelerate the healing process after experimental myocardial infarction? Int J Cardiol 2006; 107: 21-29.

10. Circi E, Akpinar S, Balcik C, Bacanli D, Guven G, Akgun RC, Tuncay IC. Biomechanical and histological comparison of the influence of oestrogen deficient state on tendon healing potential in rats. Int Orthop 2009; 33: 1461-1466.
11. Masgrau-Peya E, Lacour M, Salomon D. Topical phenytoin accelerates healing in epidermolysis bullosa simplex. Dermatology 1995; 190: 254.

12. Williams JG. Achilles tendon lesions in sport. Sports Med 1986; 3 : 114-135.

13. Sharma P, Maffulli N. Biology of tendon injury: healing, modeling and remodeling. J Musculoskelet Neuron Interact 2005; 6: 181-190.

14. Sharma $\mathbf{P}$, Maffulli $\mathbf{N}$. Tendon injury and tendinopathy: healing and repair. J Bone Joint Surg Am 2005; 87: 187-202

15. Kanaparthy R, Kanaparthy A, Devi K. Fibroblasts and phagocytic cells in phenytoin-induced connective tissue proliferation. J Orofac Res 2012; 2: 113-120.

16. Er N, Kasaboglu O, Atabek A, Oktemer K, Akkocaoglu M. Topical phenytoin treatment in bimaxillary osteomyelitis secondary to infantile osteopetrosis: report of a case. J Oral Maxillofac Surg 2006; 64: 1160-1164.

17. Muthukumarasamy MG, Sivakumar G, Manoharan G. Topical phenytoin in diabetic foot ulcers. Diabetes Care 1991; 14: 909-911.

18. Bhatia A, Nanda S, Gupta U, Gupta S, Reddy BS. Topical phenytoin suspension and normal saline in the treatment of leprosy trophic ulcers: a randomized, double-blind, comparative study. J Dermatol Treat 2004; 15: 321-327.

19. Bazin S, Delaunay A. Effect of phenytoin on the maturation of collagen in normal skin and granulomatous tissue. C R Acad Sci Hebd Seances Acad Sci. 1972; 275: 509-511.

20. Eisenberg M, Stevens LH, Schofield PJ. Epidermolysis bullosa: New therapeutic approaches. Aust J Dermatol 1978; 19: 1-8.

21. Bauer EA, Cooper TW, Tucker DR, Esterly NB. Phenytoin therapy of recessive dystrophic epidermolysis bullosa. N Engl J Med 1980; 303: 776.

22. Swamy SM, Tan P, Zhu YZ, Lu J, Achuth HN, Moochhala S. Role of phenytoin in wound healing: microarray analysis of early transcriptional responses in human dermal fibroblasts. Biochem Biophys Res Commun 2004; 314: 661-666.

23. Tengood J, Ridenour R, Brodsky RR, Russell AJ, Little S. Sequential delivery of basic fibroblast growth factor and platelet derived growth factor for angiogenesis. Tissue Eng Part A 2011; 17: 1181-1189.

24. Hong HH, Trackman PC. Cytokine regulation of gingival fibroblast lysyl oxidase, collagen, and elastin. J Periodontol 2002; 73: 145-152.

25. Okada A, Shiomi T, Aoki Y, Fujiwara M. Phenytoin stimulates chondrogenic differentiation in mouse clonal chondrogenic EC cells, ATDC5. J Toxicol Sci 2005; 30: 145-156.

Received March 7, 2016. Accepted March 20, 2016. 